from biopsy to surgery was not. Furthermore, no association between time to surgery and biochemical recurrence was seen in patients deemed to be at high risk of biochemical recurrence.

The authors conclude that, while further studies are needed to determine the impact of longer periods of time between diagnosis and surgery, their findings may help to reassure patients that taking time to make informed decisions will not have a negative impact on the success of their treatment.

Tamsin Osborne

Original article Boorjian SA et al. (2005) Does the time from biopsy to surgery affect biochemical recurrence after radical prostatectomy? BJU Int 96: 773-776

\section{Overactive bladder and interstitial cystitis}

The results of a recent retrospective study by Minaglia et al. point to a high prevalence of interstitial cystitis (IC) in women with detrusor overactivity who do not respond to anticholinergic therapy. The symptoms of overactive bladder and IC overlap, and can make it difficult for clinicians to distinguish between the two.

The team of US researchers used the potassium sensitivity test (PST) to estimate the prevalence of IC in patients with detrusor overactivity. Of the 47 women with phasic, idiopathic detrusor overactivity who were included in the study, 31 were assessed using the PST, and 25 (80.6\%) of these patients had a positive PST result. All patients had received one or more anticholinergic medications while awaiting referral to the urogynecology clinic.

A total of 25 patients did not have a satisfactory response to anticholinergic therapy, and $24(96 \%)$ of these had a positive PST result. This yields an odds ratio of $120.0(P=0.0002)$ of a positive PST result in women who did not respond to anticholinergic therapy, compared with those who did.

Based on these findings, the authors recommend that a diagnosis of IC should be considered in all women with overactive bladder who do not respond to treatment with anticholinergic medication.

Tamsin Osborne

Original article Minaglia et al. (2005) Increased prevalence of interstitial cystitis in women with detrusor overactivity refractory to anticholinergic therapy. Urology 66: 702-706

\section{Y chromosome microdeletion confers susceptibility to testicular germ cell tumor}

A large, international, multicenter study has shown that the $\mathrm{Y}$ microdeletion gr/gr confers susceptibility to testicular germ cell tumor (TGCT). This microdeletion has recently been found to be associated with infertility due to spermatogenic failure, a trait associated with TGCT disproportionately to what would be expected.

The authors analyzed this microdeletion in a series of 4,441 men recruited from familial studies, case series, case-control series, and unaffected individuals. The deletion was genotyped using multiplex polymerase chain reaction and the results were analyzed using logistic modeling.

The results showed that men with TGCT had a twofold increased risk of carrying the microdeletion, and men with TGCT and a family history of TGCT had a threefold increased risk of carrying the microdeletion. The association was stronger for seminoma $(P=0.004)$ than for nonseminoma $(P=0.29)$. Unfortunately, the TGCT patients' fertility status was not available, which prevented the authors from directly investigating the relationship between TGCT, infertility, and the gr/gr microdeletion.

All the genes affected by the microdeletion are involved in male germ-cell development and differentiation, although their functions are not completely understood. The authors conclude that the gr/gr microdeletion is a risk factor for TGCT, which might interact with other factors, either genetic or environmental, to increase susceptibility to TGCT.

Tamsin Osborne

Original article Nathanson KL et al. (2005) The Y deletion gr/gr and susceptibility to testicular germ cell tumor. $\mathrm{Am} \mathrm{J}$ Hum Genet 77: 1034-1043

\section{Promising chemotherapy regimen for hormone-refractory prostate cancer}

Tesmilifene seems to enhance the cytotoxicity of mitoxantrone for hormone-refractory prostate cancer, according to a single arm, phase II, nonrandomized clinical trial.

Hormone-refractory prostate cancer is notoriously difficult to treat. The cytotoxic 